\title{
Conocimientos y creencias sobre el trastorno por déficit de atención con hiperactividad en maestros de tres países latinoamericanos
}

\author{
Lino Palacios-Cruz, ${ }^{1}$ Francisco de la Peña Olvera, ${ }^{2}$ Gamaliel Victoria Figueroa, ${ }^{3}$ \\ Adriana Arias Caballero, ${ }^{1}$ Leysi de la Rosa Muñoz, ${ }^{3}$ Andrés Valderrama Pedroza, ${ }^{4}$ \\ Pamela Calle Portugal, ${ }^{3}$ Rosa Elena Ulloa Flores ${ }^{3}$
}

Artículo original

\section{SUMMARY}

\section{Background}

Attention deficit/hyperactivity disorder (ADHD) is a health problem that affects school functioning. To recognize the teachers' knowledge and beliefs (KB) about ADHD is important for the development of psychoeducative and training strategies for teachers. There are few Latin American reports about the teachers' $\mathrm{KB}$ and none comparing them among different countries.

\section{Objective}

To evaluate and compare the teachers' KB about ADHD in Mexico, Dominican Republic (DR) and Bolivia.

\section{Methods}

Previous verbal informed consent, the teacher's version of CASOTDAH, a self-report document that was constructed based on other instruments, was applied. The answers were examined with descriptive statistics.

\section{Results}

311 public and private school teachers were evaluated, 192 (61.7\%) from DR, 84 (27\%) from Mexico and 35 (11.3\%) from Bolivia; 79.3\% of them considered ADHD as a disease. Most of the sample considered the psychologist as the competent health professional for its diagnosis and treatment. Combined treatment was the most frequently identified as the ideal $(44.1 \%)$. Regarding their KB about the pharmacological treatment, only $14.7 \%$ identified the drug as the main component of the multimodal treatment. The teachers recognized the treatment effects on the social and academic functioning. Differences were found among teachers from the three countries regarding the importance of drug treatment or the need for multimodal treatment.

\section{Conclusions}

Teachers identify ADHD as a disease, albeit without a clear recognition of its biological components. There were differences among countries, which should be taken into account in the design of the local health psychoeducation and attention programs.
Key words: Knowledge and beliefs, teachers, ADHD, Latin America.

\section{RESUMEN}

\section{Antecedentes}

El trastorno por déficit de atención con hiperactividad (TDAH) es un problema de salud que afecta el funcionamiento escolar de quienes lo padecen. Comprender los conocimientos y creencias (CC) de los maestros resulta fundamental para el desarrollo de estrategias psicoeducativas y de capacitación para los docentes. Son pocos los reportes en Latinoamérica sobre los CC en los maestros y ninguno que compare reportes en más de un país.

\section{Objetivo}

Evaluar y comparar los CC de los maestros de niños y adolescentes en tres países latinoamericanos (México, República Dominicana [RD] y Bolivia).

\section{Método}

Previo consentimiento verbal informado, se aplicó la versión para maestros de la Cédula de Autorreporte sobre el TDAH (CASO TDAH), que fue construida a partir de otros instrumentos. Se examinaron las respuestas con estadística descriptiva y comparativa.

\section{Resultados}

Se evaluaron 311 profesores de escuelas públicas y privadas, 192 $(61,7 \%)$ de RD, 84 (27\%) de México y 35 (11.3\%) de Bolivia. El $79.3 \%$ consideró el TDAH como una enfermedad; la mayor parte de la muestra consideró al psicólogo como el profesional de salud indicado para su diagnóstico y tratamiento. El tratamiento combinado fue el más frecuentemente señalado como el ideal $(44.1 \%)$. Con respecto a sus CC acerca del tratamiento farmacológico, sólo el $14.7 \%$ señaló al fármaco como el componente más importante del tratamiento integral. Los maestros reconocieron los efectos del tratamiento en el funcionamiento social, además del académico. Sin embargo, existieron diferencias entre países con respecto al grado de impacto del mismo o la necesidad de tratamiento combinado.

Subdirección de Investigaciones Clínicas, Instituto Nacional de Psiquiatría Ramón de la Fuente Muñiz.

Departamento de Fomento a la Investigación, Instituto Nacional de Psiquiatría Ramón de la Fuente Muñiz.

3 Psicofarmacología del Desarrollo, Hospital Psiquiátrico Infantil Juan N. Navarro, México, DF.

4 Universidad Autónoma de San Luis Potosí, Coordinación del Curso de Posgrado en Psiquiatría/Psiquiatría Infantil y de la Adolescencia.

Correspondencia: Dra. Rosa Elena Ulloa Flores. Psicofarmacología del Desarrollo, Hospital Psiquiátrico Infantil Juan N. Navarro, San Buenaventura 86, Belisario Domínguez, Tlalpan, 14080, México, DF. E.mail:eulloa@hotmail.com

Recibido primera versión: 13 de diciembre de 2012. Segunda versión: 6 de marzo de 2013. Aceptado: 9 de abril de 2013 


\section{Conclusiones}

Los maestros identificaron al TDAH como una enfermedad, aunque el reconocimiento de sus aspectos biológicos no fue claro. Existen diferencias por país que deben ser tomadas en cuenta en los diseños de los programas locales de atención a la salud.
Palabras clave: Conocimientos y creencias, maestros, TDAH, Latinoamérica.

\section{INTRODUCCIÓN}

El trastorno por déficit de atención con hiperactividad (TDAH) es un padecimiento del neurodesarrollo que se inicia durante la infancia y cuyos principales síntomas son la inatención, la hiperactividad y la impulsividad. ${ }^{1}$ El TDAH es el trastorno externalizado más frecuente en población pediátrica, su prevalencia acumulada a nivel mundial es de $5.29 \%{ }^{2}$ Los niños y adolescentes con TDAH tienen mayor riesgo de enfrentar dificultades académicas y de padecer trastornos afectivos, ansiosos y de conducta. La etiología de este trastorno incluye factores neurobiológicos y medioambientales. ${ }^{3}$ Las investigaciones en neurociencias, los estudios genéticos ${ }^{4}$ y los ensayos farmacológicos ${ }^{5}$ han aportado distintos datos que apoyan en mayor medida su origen biológico.

Fuera del ámbito médico, el TDAH resulta controvertido en su diagnóstico y tratamiento. Muchas de las críticas apuntan a que no es un trastorno real sino más bien una constelación de conductas que los padres y los maestros no están dispuestos a enfrentar o no saben cómo manejar. ${ }^{6}$

Los maestros cumplen un papel esencial al momento de evaluar problemas conductuales y de rendimiento académico. ${ }^{7}$ Son además una fuente valiosa de información debido a que tienen contacto con los niños y adolescentes en varias situaciones y con frecuencia son los primeros en canalizarlos para una evaluación diagnóstica. Los sujetos con TDAH grave son los más frecuentemente referidos. ${ }^{8,9}$ Dada la importancia de la información de los maestros, el DSM-5 requerirá la opinión de estos profesionales para establecer el diagnóstico de TDAH. ${ }^{10}$ Así como son importantes al momento de la evaluación clínica, los maestros desempeñan un papel fundamental en el tratamiento del TDAH. Kauffman et al. ${ }^{11}$ mostraron en un estudio con 61 maestros que la mayoría de ellos se creían capaces de enseñar habilidades críticas a los estudiantes, como escuchar y seguir las reglas en el salón de clase, y de manejar conductas inaceptables como los berrinches y el robo. A pesar de ello, el porcentaje de maestros integrados en los procesos diagnósticos y terapéuticos de sus alumnos con TDAH es bajo. ${ }^{12} \mathrm{El}$ manejo clínico incluye la participación de los maestros, los padres y el tratamiento farmacológico, siendo este último la piedra angular del mismo. ${ }^{13,14} \mathrm{El}$ objetivo del tratamiento no es sólo la reducción de síntomas, sino también la recuperación de la funcionalidad. . $^{15,16}$

La participación de los profesionales de la salud y de la educación en las distintas fases de la evaluación clínica y del tratamiento de los niños y adolescentes con TDAH hace necesario investigar las creencias, conocimientos y actitudes de los profesionales de la educación que tratan con niños y adolescentes con este padecimiento. La mayor parte de los estudios conducidos durante los últimos 15 años se han realizado en países de habla inglesa como Estados Unidos y Australia.9,12,17-

${ }^{20}$ Estos estudios señalaron que la información científica en esta área es limitada; por ejemplo, el porcentaje de respuestas correctas en un cuestionario sobre conocimientos acerca del TDAH va de 47.8 a $77.5 \% .{ }^{9,12,17,20} \mathrm{Al}$ parecer, el nivel de experiencia laboral no influye en los mitos relacionados con el TDAH, como el de los aditivos en los alimentos como causa del trastorno. ${ }^{18}$ Además, los maestros en servicio no reconocieron más el TDAH que los que estaban en formación. ${ }^{19}$

Al evaluar a 87 maestros turcos de escuelas primarias, se observó que sus conocimientos acerca del origen del TDAH fueron relativamente escasos: $32.2 \%$ señalaron que el TDAH se debe a vulnerabilidades biológicas y causas genéticas, y $65.5 \%$ contestaron que es consecuencia de una mala crianza. El $67.8 \%$ reconoció que los niños con TDAH experimentan disfunción social, además de la académica. ${ }^{21}$

Existen algunas diferencias entre los conocimientos y creencias de los maestros de niños en edad escolar y los maestros de adolescentes. Un estudio en 193 maestros españoles de educación primaria mostró que tenían mayor conocimiento sobre los síntomas y el diagnóstico que sobre el tratamiento del TDAH. ${ }^{22}$ A su vez, una reciente investigación en el sureste de Estados Unidos demostró que los maestros de adolescentes con TDAH estaban dispuestos a que los alumnos recibieran tratamiento psicosocial y farmacológico. ${ }^{23}$

La información acerca de los conocimientos y creencias de los maestros sobre el TDAH en Latinoamérica es escasa. Un estudio realizado en Puerto Rico evaluó los conocimientos sobre la enfermedad en 132 maestros de escuelas públicas y privadas, donde $35 \%$ reportó tener conocimientos previos respecto al TDAH y $72 \%$ mostró un nivel bajo de conocimientos. ${ }^{24}$ No existen estudios que contrasten poblaciones de maestros en el subcontinente latinoamericano.

El objetivo de esta investigación fue describir y comparar los conocimientos y creencias sobre el TDAH en los maestros de niños y adolescentes de México, Bolivia y República Dominicana (RD).

\section{MATERIAL Y MÉTODOS}

La muestra fue conformada por maestros de nivel preescolar a preparatoria y de escuelas públicas y privadas. Todos los maestros accedieron a responder la cédula de autorre- 
porte sobre el TDAH (CASO TDAH), versión Maestros. El procedimiento de reclutamiento fue directo en las escuelas y por conveniencia. El proyecto cumplió con los requisitos establecidos por la Declaración de Helsinki y fue aprobado por los comités de ética correspondientes.

\section{Instrumentos}

CASO TDAH. Esta cédula es un cuestionario construido a partir de otros instrumentos. ${ }^{25,26}$ Tiene tres versiones: a) padres, b) maestros y c) profesionales de la salud. La que se reporta en este estudio es la versión que se aplicó a los maestros, que consiste en un cuestionario autoaplicable compuesto por 21 preguntas de opción múltiple relacionadas con cuatro áreas: a) lugar de desempeño laboral, b) conocimientos acerca del TDAH, c) creencias acerca del diagnóstico y tratamiento y d) manejo de niños con problemas de atención y conducta. El cuestionario se responde en un tiempo promedio de 20 minutos.

\section{Análisis estadístico}

Los resultados se analizaron con el programa PASW Statistics 18. Cuando hubo de 5 a $15 \%$ de datos faltantes en alguna variable, se uso el método de imputación simple para la sustitución de datos. ${ }^{27}$ Para la descripción de los resultados, se utilizaron medidas de tendencia central y de dispersión. Para las comparaciones entre variables nominales, se empleó $\mathrm{Chi}^{2}$ de tendencia lineal. Se consideró significativo un valor de $\mathrm{p}<0.05$.

\section{RESULTADOS}

Se evaluaron 311 maestros: 192 (61.7\%) de RD, 84 (27\%) de México y 35 (11.3\%) de Bolivia. El 55.5\% (N=172) laboraba en escuelas públicas y el resto en privadas. El 50.8\% (N=158) trabajaba con adolescentes, $40.8 \%(\mathrm{~N}=127)$ con escolares y $8 \%(\mathrm{~N}=25)$ con preescolares; uno de los encuestados mencionó trabajar con adultos. No se encontraron diferencias en cuanto al tipo de escuela ni al grupo etáreo de los alumnos de estos maestros. El 28.8\% ( $\mathrm{N}=85)$ de los maestros tenía de uno a dos alumnos y el $40.3 \%(\mathrm{~N}=119)$ entre tres y cinco alumnos con problemas académicos o de conducta.

\section{Conocimientos acerca del TDAH}

El 87.3\% $(\mathrm{N}=268)$ de los maestros dijo saber qué era el TDAH, opinando de la siguiente manera: $79.3 \%(\mathrm{~N}=237)$ respondió que era una enfermedad, $17.7 \%(\mathrm{~N}=53)$ respondió que era un problema de crianza y $1 \%$ respondió que era un mito, una moda o un invento de la industria farmacéutica.

Los conocimientos de los maestros respecto al impacto de la enfermedad y los riesgos asociados a ella mostraron diferencias por país: a) los maestros de RD consideraban con mayor frecuencia que los niños y adolescentes con TDAH tenían mayor riesgo para iniciar vida sexual antes de los 18 años $(90.4 \%$ vs. $76.5 \%$ y $66.7 \%$ de México y Bolivia, respectivamente; $\left.\chi^{2}=11.445, \mathrm{gl}=1, \mathrm{p}=0.001\right)$; b) los maestros de Bolivia consideraban que tenían mayor riesgo para fracturas o lesiones graves $(93.3 \%$ vs. $85.1 \%$ y $67.5 \%$ de México y RD respectivamente, $\left.\chi^{2}=9.862, \mathrm{gl}=1, \mathrm{p}=0.002\right)$; c) los maestros de México consideraron que tenían más accidentes $(89.7 \%$ vs. $87.5 \%$ y $75.9 \%$ de Bolivia y RD, respectivamente, $\mathrm{X}^{2}=4.881$, $\mathrm{gl}=1, \mathrm{p}=0.02)$.

\section{Conocimientos y creencias acerca del diagnóstico y tratamiento}

La mayor parte de la muestra consideró que el psicólogo era el profesional capacitado para diagnosticar y tratar esta condición (cuadro 1). En la comparación por países, los maestros de RD mencionaron que el psicólogo era el profesional capacitado para hacer el diagnóstico con mayor frecuencia (67.7\% vs. $54.8 \%$ y $48.6 \%$ de México y Bolivia, respectivamente, $\chi^{2}=6.946, \mathrm{gl}=1, \mathrm{p}=0.0008$ ); un mayor porcentaje de maestros de RD señaló al psicólogo como el profesional más adecuado para tratar esta condición (58.6\% vs. 51.4\% y 41.2 de México y Bolivia, respectivamente, $\chi^{2}=3.951$, gl=1, $\mathrm{p}=0.04$ ), mientras que un mayor porcentaje de los maestros de Bolivia consideró a los neurólogos o neuropediatras ( $14.7 \%$ vs. $5.78 \%$ y $4.7 \%$ de México y RD, respectivamente, $\mathrm{X}^{2}=3.829, \mathrm{gl}=1, \mathrm{p}=0.05$ ) como adecuados para tratar a los pacientes con TDAH.

\section{Tipos de tratamiento}

Los maestros señalaron diversos tipos de tratamiento tanto farmacológicos como psicosociales; la figura 1 muestra los más frecuentemente señalados como ideales para el TDAH.

En relación con los conocimientos sobre los medicamentos, el 55.6\% ( $\mathrm{N}=155)$ consideró que sólo servían como apoyo al tratamiento psicológico o pedagógico; $14.7 \%(\mathrm{~N}=41)$ señaló que constituían la parte más importante dentro del tratamiento integral y $9.7 \%(\mathrm{~N}=27)$ refirió que no tenían utilidad. El 14\% (N=39) mencionó que los fármacos produ-

Cuadro 1. Profesionales capacitados para diagnosticar y tratar a niños y adolescentes con TDAH de acuerdo a los maestros de México, Bolivia y República Dominicana

\begin{tabular}{lcc}
\hline & $\begin{array}{c}\text { Diagnóstico } \\
{[\mathrm{N}=311] \%}\end{array}$ & $\begin{array}{c}\text { Tratamiento } \\
{[\mathrm{N}=295] \%}\end{array}$ \\
\hline Psiquiatra/Psiquiatra Infantil & 37.6 & 10.8 \\
Neurólogo/Neurólogo Pediatra & 26.7 & 6.1 \\
Psicólogo & 62.1 & 54.9 \\
Neuropsicólogo & 25.7 & 0.0 \\
Pedagogo & 50.8 & 12.2 \\
Pediatra & 5.5 & 0.0 \\
Psicoterapeuta & 0.0 & 25.4 \\
\hline
\end{tabular}




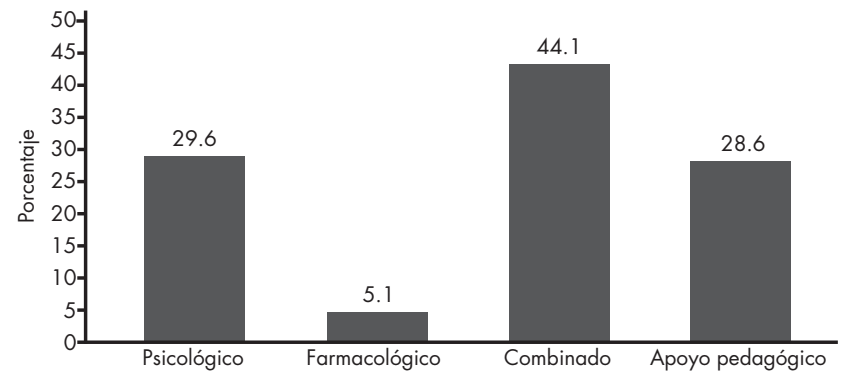

Figura 1. Tratamiento ideal para una persona con TDAH.

cían efectos secundarios y $8.2 \%(\mathrm{~N}=23)$ dijo que provocaban adicción.

Una alta proporción de los maestros evaluados consideró que el tratamiento mejoraba: el funcionamiento académico, 64.3\% ( $\mathrm{N}=153)$; las relaciones sociales, 53.4\% ( $\mathrm{N}=127)$, el apego a la rutina del colegio, 51.7\% ( $\mathrm{N}=123)$, y las actividades de tiempo libre, 37\% ( $\mathrm{N}=88)$.

Las diferencias en los conocimientos y creencias sobre el tratamiento por país se muestran en el cuadro 2.

\section{Conductas de los maestros ante los alumnos con TDAH}

El 82.5\% ( $\mathrm{N}=255)$ mencionó tener experiencia en el manejo de niños y adolescentes con problemas de conducta dentro del aula de clases, y el 59.7\% (N=181) señaló tener experiencia en el manejo del TDAH.

El 42.1\% (N=130) utilizaba medidas de manejo conductual dentro del salón de clases al detectar alumnos con problemas de conducta o de rendimiento escolar. Además de estas estrategias, $8 \%(\mathrm{~N}=25)$ notificaba a los padres que sus hijos tenían problemas de conducta y $0.9 \%(\mathrm{~N}=3)$ solicitaba valoración por un profesional de la salud. Sólo el $4.2 \%$ $(\mathrm{N}=13)$ de los maestros incluía en sus intervenciones a los padres y al profesional de la salud, además del manejo dentro del salón de clases. Al comparar los países, se encontró que los maestros de RD avisaban a los padres al detectar algún problema de conducta en los alumnos en mayor proporción que los de Bolivia y México (44.8\% vs. $28.6 \%$ y 26.2\%, respectivamente, $\left.\chi^{2}=7.736, g l=1, p=0.005\right)$. También reportaron con mayor frecuencia sentarlos al frente y supervisarlos de cerca comparados con los de México y Bolivia (27.1\% vs. $11.9 \%$ y $11.4 \%$, respectivamente, $\left.\chi^{2}=8.699, \mathrm{gl}=1, \mathrm{p}=0.003\right)$.

\section{DISCUSIÓN}

El presente trabajo describe y compara los conocimientos y creencias de maestros de tres diferentes países latinoamericanos, que representan a Norteamérica, Sudamérica y el Caribe. La muestra estudiada fue proporcional en cuanto al centro educativo público o privado; el 90\% trabajaba con escolares o adolescentes. La distribución por país no fue equitativa ya que los maestros de RD representaron el 61.7\%. Es importante hacer notar que casi $70 \%$ de los maestros encuestados refirieron tener al menos un alumno con problemas de aprovechamiento o conducta. Esto es similar a reportes previos ${ }^{28}$ y muestra que la población estudiada tenía contacto cotidiano con esta psicopatología.

El porcentaje de maestros que señaló al TDAH como una enfermedad (79\%) fue similar a los resultados de algunos estudios realizados por profesionales no médicos, ${ }^{28}$ lo que sugiere que nuestros resultados no están influidos por el hecho de que las cédulas fueran distribuidas por un equipo médico. Por otro lado, este porcentaje es mayor al reportado en estudios de países como Irán y Sri Lanka, donde más de la mitad de los maestros encuestados consideró al TDAH como un problema de crianza. . $9,30^{29}$

En el presente estudio, la mayor parte de los maestros consideraba al psicólogo como el profesional de elección para diagnosticar y tratar el TDAH. Estudios previos mostraron que los maestros no tienen contacto regular con médicos que tratan este padecimiento. ${ }^{17} \mathrm{Al}$ respecto, vale la pena mencionar que en los países del presente estudio

Cuadro 2. Diferencias en los conocimientos y creencias sobre el tratamiento entre los maestros de Bolivia, México y RD

\begin{tabular}{|c|c|c|c|c|c|c|}
\hline & $\begin{array}{l}\text { Bolivia } \\
(\mathrm{N}=35) \%\end{array}$ & $\begin{array}{l}\text { México } \\
(\mathrm{N}=84) \%\end{array}$ & $\begin{array}{c}\text { República } \\
\text { Dominicana } \\
(\mathrm{N}=192) \%\end{array}$ & $x^{2}$ & $g l$ & $p$ \\
\hline $\begin{array}{l}\text { El tratamiento ideal para una persona que padece TDAH es el } \\
\text { tratamiento psicológico. }\end{array}$ & 14.3 & 17.9 & 37.5 & 13.686 & 1 & 0.000 \\
\hline $\begin{array}{l}\text { El tratamiento ideal para una persona que padece TDAH incluye } \\
\text { el tratamiento psicológico y farmacológico. }\end{array}$ & 45.7 & 63.1 & 35.4 & 8.062 & 1 & 0.005 \\
\hline $\begin{array}{l}\text { El tratamiento farmacológico sólo sirve como apoyo al trata- } \\
\text { miento psicológico o pedagógico. }\end{array}$ & 31.4 & 60.3 & 58.5 & 5.394 & 1 & 0.02 \\
\hline $\begin{array}{l}\text { El tratamiento farmacológico es la parte más importante dentro } \\
\text { del tratamiento integral. }\end{array}$ & 25.7 & 22.1 & 9.7 & 9.204 & 1 & 0.002 \\
\hline La rutina normal mejora con el tratamiento. & 33.3 & 50.7 & 56.6 & 5.225 & 1 & 0.02 \\
\hline El tratamiento mejora las relaciones sociales. & 33.3 & 55.1 & 57.4 & 4.660 & 1 & 0.03 \\
\hline
\end{tabular}


existe un mayor número de psicólogos que de neurólogos y psiquiatras (hasta 100 veces más). ${ }^{31-34} \mathrm{Al}$ ser el psicólogo el profesional de la salud mental con mayor disponibilidad en estos tres países, probablemente sea quien más influya en la información que reciben los maestros sobre el diagnóstico y tratamiento del TDAH. Las investigaciones que estudiaron las creencias de los psicólogos sobre este padecimiento han señalado que lo consideraban una enfermedad con un alto componente psicosocial, susceptible de ser modificada con medidas ambientales. ${ }^{35}$ En este sentido, cabe señalar que sólo el $44.1 \%$ de los maestros expresó que el tratamiento ideal es el combinado y 55.6\% señaló que el medicamento sirve como apoyo al tratamiento psicológico o pedagógico. Lo anterior sugiere una falta de información sobre el sustrato neurobiológico de este padecimiento y la necesidad de tratamiento farmacológico, como se ha visto en otros países. ${ }^{36-38}$ Además de esto, es importante hacer notar que aunque la mayor parte de los maestros encuestados se consideraban capaces de manejar a un niño con TDAH en el salón de clases, pocos consideraban incluirse en un manejo conjunto con los padres y profesionales de la salud, lo que también apunta a la necesidad de educar a los maestros acerca de su papel dentro de un esquema de manejo multimodal.

La proporción de maestros que señalaron que el tratamiento combinado era el ideal fue similar a la reportada en un estudio realizado en España. ${ }^{22}$ En el presente estudio los maestros de México señalaron en mayor proporción que el tratamiento ideal era el tratamiento combinado. A su vez, los maestros bolivianos identificaron en menor proporción que el tratamiento farmacológico ayudaba a los tratamientos psicológicos y pedagógicos. Esto último se asemeja a los resultados de un estudio realizado en Estados Unidos, donde, a pesar de recibir entrenamiento específico acerca del TDAH, 34\% de los maestros consideraban el uso de estimulantes como un último recurso de tratamiento. ${ }^{17}$ Estas diferencias resaltan la necesidad de desarrollar estrategias psicoeducativas y de capacitación específicas para cada país.

Aunque en general los maestros de los tres países pudieron identificar el impacto del TDAH en la vida social y la seguridad de sus alumnos, se apreciaron ciertas diferencias. Así, los maestros de Bolivia reportaron en menor porcentaje que el TDAH conlleva mayor riesgo para el inicio temprano de la vida sexual y los de RD para sufrir accidentes. Estudios previos mencionan que los maestros tienden a estar más preocupados por las conductas problemáticas de sus alumnos que por sus dificultades sociales. ${ }^{11}$

Aunque Latinoamérica tiene, como región, características comunes como la existencia de una mayor proporción de psicólogos que de psiquiatras y la conceptualización del TDAH como enfermedad, las diferencias por país apuntan a necesidades específicas que deben tomarse en cuenta para diseñar programas de psicoeducación y atención integral del trastorno.

\section{Limitaciones}

Es importante tomar en cuenta los siguientes puntos: La muestra no fue obtenida al azar sino por conveniencia, siendo diferente para cada país, lo que resta representatividad y validez externa a los resultados obtenidos a partir de esta población. No se investigaron los años de experiencia laboral de los maestros ni la familiaridad del maestro con el trastorno, ya sea por tener un pariente con este padecimiento o por haber recibido información o capacitación previa. La literatura científica no ha mostrado que la experiencia como maestros ni la edad estén relacionadas con sus conocimientos sobre el TDAH. ${ }^{24,28}$ Otro punto que debe ser tomado en cuenta es que la información no fue obtenida por medio de una escala, sino por un cuestionario expresamente diseñado para este estudio con la incorporación de información de instrumentos aplicados en padres o en profesionales de la salud. Este cuestionario trató de explorar distintos aspectos por medio de preguntas de opción múltiple o abiertas con una respuesta concreta, las cuales fueron asignadas a un área (conocimientos, creencias o conductas) a juicio de los investigadores, lo que constituyó su validez aparente.

\section{CONCLUSIONES}

Los maestros identificaron al TDAH como una enfermedad, aunque no claramente a su componente biológico y por consiguiente la necesidad de tratamiento farmacológico, por lo que es recomendable mejorar la comunicación entre los profesionales de la salud y los maestros. Aunque muchas respuestas fueron similares, hubo diferencias por país. Éstas deben tomarse en cuenta al momento de diseñar los programas locales de psicoeducación y atención al TDAH.

\section{AGRADECIMIENTOS}

Los autores agradecen a Jorge Macías Garza y a Gabriela López su colaboración en la edición del manuscrito.

\section{REFERENCIAS}

1. American Psychiatric Association. Diagnostic and Statistical Manual of Mental Disorders. Cuarta edición (DSM-IV). Washington, DC: 1994.

2. Polanczyk G, Silva de Lima M, Lessa Horta B, Biederman J et al. The worldwide prevalence of ADHD: A systematic review and metaregression analysis. Am J Psychiatry 2007;164(6):942-948.

3. Steinhausen $\mathbf{H}$. The heterogeneity of causes and courses of attentiondeficit/hyperactivity disorder. Acta Psychiatr Scand 2009;120(5):392-399.

4. Doyle A, Faraone S, Seidman L, Willcutt EG, Nigg JT, Waldman ID, Pennington BF, Peart J, Biederman J. Are endophenotypes based on measures of executive functions useful for molecular genetic studies of ADHD? J Child Psychol Psychiatry 2005;46(7):774-803.

5. Faraone S, Biederman J, Spencer T, Aleardi M. Comparing the efficacy of medications for ADHD using meta-analysis. MedGenMed 2006;8(4):4. 
6. McLeod J, Fettes D, Jensen P, Pescosolido B et al. Public knowledge, beliefs, and treatment preferences concerning attention deficit- hyperactivity disorder. Psychiatr Serv 2007;58(5):626-631.

7. Stevens J, Quittner A, Abikoff H. Factors influencing elementary school teachers' ratings of ADHD and ODD behaviors. J Clin Child Psychol 1998;27(4):406-414.

8. Weyandt L, Fulton K, Schepman S, Verdi G et al. Assessment of teacher and school psychologist knowledge of attention-deficit/hyperactivity disorder. Psychol Sch 2009;46(10):951-961.

9. Sciutto $M$, Terjesen $M$, Bender Frank A. Teachers' knowledge and misperceptions of Attention-Deficit/Hyperactivity disorder. Psychol Sch 2000;37(2):115-122.

10. American Psychiatric Association. DSM-5: Options Being Considered for ADHD. 2010. Consultado en: http://www.dsm5.org/Proposed\%20R evision\%20Attachments/APA\%20Options\%20for\%20ADHD.pdf el 02 febrero 2012.

11. Kauffman J, Lloyd J, McGee K. Adaptive and maladaptive behavior: Teachers' attitudes and their technical assistance needs. J Spec Educ 1989;23(2):185-200.

12. Jerome L, Gordon M, Hustler P. A comparison of American and Canadian teachers' knowledge and attitudes towards Attention Deficit Hyperactivity Disorder (ADHD). Can J Psychiatry 1994;39(9):563-567.

13. Biederman J, Spencer T, Wilens T. Evidence-based pharmacotherapy for attention-deficit hyperactivity disorder. Int J Neuropsychopharmacol 2004;7(1):77-97.

14. Pelham WJ, Fabiano G. Evidence-based psychosocial treatments for attention-deficit/hyperactivity disorder. J Clin Child Adolesc Psychol 2008;37(1):184-214.

15. De la Peña F, Barragán E, Rohde L, Patiño LR et al. Algoritmo de tratamiento multimodal para escolares latinoamericanos con trastorno por déficit de atención con hiperactividad (TDAH). Salud Mental 2009;32:17-29.

16. Swanson J, Arnold L, Kraemer H, Hechtman L et al. MTA Cooperative Group. Evidence, interpretation, and qualification from multiple reports of long-term outcomes in the Multimodal Treatment Study of children with ADHD (MTA): Part II: supporting details. J Atten Disord 2008;12(1):15-43.

17. Barbaresi W, Olsen R. An ADHD educational intervention for elementary schoolteachers: a pilot study. J Dev Behav Pediatr 1998;19(2):94-100.

18. Bekle B. Knowledge and attitudes about attention-deficit hyperactivity disorder (ADHD): a comparison between practicing teachers and undergraduate education students. J Atten Disord 2004;7(3):151-161.

19. Jerome L, Washington $P$, Laine $C$, Segal A. Graduating teachers' knowledge and attitudes about attention-deficit hyperactivity disorder: a comparison with practicing teachers. Can J Psychiatry 1999;44(2):192.

20. Kos J, Richdale A, Jackson M. Knowledge about Attention-Deficit/Hyperactivity Disorder: A comparison of in-service and preservice teachers. Psychol Sch 2004;41(5):517-526.

21. Nur N, Kavakc O. Elementary school teachers' knowledge and attitudes related to attention deficit hyperactivity disorder. HealthMED 2010;4(2):350-355.

22. Jarque Fernández S, Tárraga Mínguez R, Miranda Casas A. [Teachers' knowledge, misconceptions, and lacks concerning Attention Deficit Hyperactivity Disorder]. Psicothema 2007;19(4):585-590.

23. Bussing R, Koro-Ljungberg M, Noguchi K, Mason D et al. Willingness to use ADHD treatments: a mixed methods study of perceptions by adolescents, parents, health professionals and teachers. Soc Sci Med 2012;74(1):92-100.

24. González Tejera G, González M, Ramírez B, Rivera M. Attention deficit hyperactivity disorder in five schools of the San Juan metropolitan area: assesment of teacher's knowledge. Bol Asoc Med P R 2009;101(1):31-35.

25. Barkley R, Murphy K, Bauermeister J. Trastorno por déficit de atención e hiperactividad. Un manual de trabajo clínico. Londres: The Guilford Press; 1998.

26. Palacio J, Botero D, Muñoz C, Vásquez R. Hallazgos de una encuesta sobre la experiencia del manejo clínico del TDAH. Rev Colomb Psiquiatr 2009;38:159S-177S.

27. Medina F, Galván M. Imputación de datos: teoría y práctica. Serie Estudios Estadísticos y Prospectivos 54. Santiago de Chile: Publicación de las Naciones Unidas; 2007. Consultado en: http:/www.eclac.org/ publicaciones/xml/9/29949/LCL2772e.pdf el 04 mayo 2012.

28. Kos J. What do teachers know, think and intend to do about ADHD? 2008. Consultado en: http://research.acer.edu.au/tll_misc/9 el 20 febrero 2013.

29. Ghanizadeh A, Bahredar M, Moeini S. Knowledge and attitudes towards attention deficit hyperactivity disorder among elementary school teachers. Patient Educ Couns 2006;63(1-2):84-88.

30. Rodrigo MD, Perera D, Eranga VP, Williams SS et al. The knowledge and attitude of primary school teachers in Sri Lanka towards childhood attention deficit hyperactivity disorder. Ceylon Med J 2011;56(2):51-54.

31. Consejo Mexicano de Neurología. Certificación vigente. 2011. Consultado en: http://www.consejomexicanodeneurologia.org/descargas/vigentes2012.pdf el 12 junio de 2012.

32. Informe sobre el sistema de salud mental en República Dominicana, Organización Panamericana de la Salud, Organización Mundial de la Salud. Informe de la evaluación del sistema de salud mental en República Dominicana utilizando el instrumento de evaluación para el sistema de salud mental de la OMS(IESM-OMS): SESPAS: OPS/OMS; 2008. Consultado en: http://www.who.int/mental_health/dominican_ republic_who_aims_spanish.pdf el 23 de julio del 2012.

33. Lara Muñoz M, Fouilloux $C$, Arévalo Ramírez M, Santiago Ventura Y. ¿Cuántos somos? ¿Dónde estamos? ¿Dónde debemos estar? El papel del psiquiatra en México. Análisis preliminar. Salud Mental 2011;34(6):531-536.

34. Rivera Arroyo G. Panorama de la salud mental en Bolivia. Consultado en: $h t t p: / / w w w . g o o g l e . c o m . m x / u r l ? s a=t \& r c t=j \& q=\& e s r c=s \& s o u r c e=w$ eb\&cd $=1 \&$ sqi $=2 \& v e d=0$ CEoQFjAA\&url $=$ http $\% 3 \mathrm{~A} \% 2 \mathrm{~F} \% 2 \mathrm{Fwww} . \mathrm{psi}-$

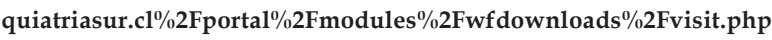
el 04 junio de 2012.

35. Victoria G, Ulloa R, Palacios-Cruz L. Conocimientos y creencias de los psicólogos sobre el trastorno por déficit de atención con hiperactividad (TDAH). Mexico Quarterly Review 2011;2(6):9-20.

36. Al-Sharbati M, Al-Sharbati Z, Al-Lawatiya S, Al-Jahwari S. Teachers' awareness about attention deficit hyperactivity disorder (ADHD) in Oman. Asian J Psychiatr 2012;5(3):277-278.

37. Brook U, Watemberg N, Geva D. Attitude and knowledge of attention deficit hyperactivity disorder and learning disability among high school teachers. Patient Educ Couns 2000;40(3):247-252.

38. Lien MT, Carlson JS, Hunter-Oehmke S, Knapp KA. A pilot investigation of teachers' perceptions of psychotropic drug use in schools. J Atten Disord 2007;11(2):172-177.

Artículo sin conflicto de intereses 